\title{
Probleme der nationalen und regionalen Identität im Sudan
}

\author{
Von Abdel Bagi A. G. Babiker
}

Seit einigen Jahrzehnten erleben die afrikanischen Länder einen tiefgreifenden Entwicklungsprozeß im Sozialleben, der stark von der Wirtschaftsentwicklung beeinflußt wird. Der materiell-technische Fortschritt bringt viele Veränderungen in der Gesellschaft und in der eigenen Kultur mit sich, so daß man von einer Identitätsbeeinflussung oder einem -verlust spricht. Diese Spannung, die durch die Konfrontation zwischen alten und modernen Werten ausgelöst wird, existiert sowohl im politischen als auch im geographischen Raum. Einige Afrikaner sind sich dieser Veränderungen bewußt und hoffen, daß ihr Bewußtsein Einfluß haben wird, um zukünftige wirtschaftliche und gesellschaftliche Veränderungen ihres Lebens mitzubestimmen. Bei dem größten Teil der Afrikaner jedoch läuft dieser Prozeß allmählich und unbewußt ab, d. h. in Form unbewußter Verhaltensänderungen. Das resultiert am Ende in einer breiten Skala von Identitätsvorgängen; sie reichen von Identitätsfindung und Identitätsäußerung, bis zum Identitätsverlust. In vielen Fällen überschneiden sich diese Prozesse, so daß man kaum in der Lage ist, einen einzelnen für sich zu erkennen.

Leider ist die Erforschung dieser wichtigen Problematik von den Geographen bisher sehr vernachlässigt worden. Dieser Beitrag, der sich mit einigen Fragen der nationalen und regionalen Identität im Sudan beschäftigt, soll einer allgemeinen Einführung dienen. Ich hoffe aber, daß er die zukünftige Erforschung dieser Frage anregen wird. Die theoretische Behandlung der Identitätsfrage liegt außerhalb des Rahmens dieses Aufsatzes. Darüber liegt genügendes Material in der Ethnologie und anderen Sozialwissenschaften vor. Es ist eine Tatsache, daß der Prozeß der Identitätssuche, -findung, -äußerung bzw. -modifikation und des -verlustes erst durch Kontakte und Beziehungen mit anderen Kulturen und Lebensarten ausgelöst wird. In einer Gesellschaft mit vielen ethnischen Gruppen werden diese Vorgänge durch die soziale Stellung der einen oder anderen Gruppe im gesamten hierarchischen System beeinflußt. Je intensiver die Beziehungen und Kontakte zwischen den Völkergruppen und ihren Kulturen sind, um so wichtiger wird die Identitätsfrage. Deshalb spielen dabei Kontaktzonen und Treffpunkte verschiedener ethnischer Gruppen eine bedeutende Rolle. Beispiele dafür sind im Sudan und auch in vielen anderen afrikanischen Ländern Verkehrsstraßen, tägliche und Wochenmärkte, Wasserstellen, Städte und administrative, also zentrale Orte.

Der Kontakt wird sicher beschleunigt durch die drastische Entwicklung der Kommunikationsmedien: Transistorradios, Schulen, Händler und Lastwagenfahrer als Informationsübermittler. Sie übermitteln Ideen und Bilder verschiedener Lebensweisen, welche die Einstellung der verschiedenen Mitglieder gegenüber ihrer Gesellschaft beeinflussen. Von Einfluß ist auch die zum Teil durch Migration verursachte schnellere Urbanisie- 
rung, die im ganzen Afrika in unterschiedlichem Maße anwächst. Viele, zum größten Teil junge Männer, wandern aus ökonomischen, sozialen und ökologischen Gründen in die Städte. Ihre Abwesenheit führt zur Veränderung der Moralvorstellungen auf dem Lande und wirkt sich auf das ganze Sozial- und Wirtschaftssystem aus. Die Wirksamkeit der großen Städte besteht nicht nur in der Mischung verschiedener Kulturen, sondern auch in der Akkumulation von Kapital und Technik, die auch in Afrika die Grundlage für alle Instrumente der Sozial- und Wirtschaftsentwicklung, wie Industrien, Schulen und Kommunikationsmedien bilden (TESSLER 1973). Neue Ideen und Interessen im Hinblick auf Konsum- und Kaufgewohnheiten, verschiedene Einstellung zur Arbeit, zu sozialen Bindungen und Beziehungen bewirken große Veränderungen in Moral und Tradition und derart in der kulturellen Identität. Städtisches Leben ermöglicht individuelle Entscheidungen und damit Veränderungen, bedingt durch monatliche und halbmonatliche Gehaltszahlungen, während auf dem Lande Entscheidungen auf der Ebene einer großen Familie oder der Sippe getroffen werden.

Nicht nur die individuelle, Gruppen- oder regionale Identität, sondern auch die nationale Identität in afrikanischen Ländern durchläuft gegenwärtig einen tiefgreifenden Veränderungsprozeß. Einerseits bemühen sich Politiker und Staatsführer, eine bestimmte Identität zu entwickeln und durchzusetzen, die einigen regionalen Identitäten nicht angepaßt ist. Deshalb versuchen sie, regionale Besonderheiten zu beseitigen. In solchen Fällen handelt es sich meistens um den Versuch, die Identität der Peripherie an die des Zentrums anzukoppeln. Andererseits entdeckten einige afrikanische Politiker die Kraft der sozialkulturellen Tradition. Sie sind gewahr geworden, daß die wirtschaftliche und soziale Entwicklung durch die Unterstützung traditioneller Werte und Organisationen schneller vor sich geht. Bei den Bemühungen solcher nationalen Identitätssuche verstärkt sich die regionale Identität. Ansätze dieser Prozesse findet man im Sudan. Uber einige davon möchte ich, im Sinne meiner Erkenntnisse interpretiert, in diesem Aufsatz berichten.

\section{Die nationale Identität im Sudan}

Bei der Betrachtung der Frage der nationalen Identität im Sudan muß man einige wesentliche Merkmale anführen, die sich positiv oder negativ auf die Identität auswirken. Die heutigen Staatsgrenzen des Sudan sind ein modernes Phänomen (seit 1916).

Wie bei vielen anderen afrikanischen Ländern wurden sie durch koloniale Willkür gezogen. In der jungen Geschichte des Sudan änderten sich die Grenzen mehrmals, so daß einige Teile und dabei Völkergruppen für längere Zeit außerhalb des Staates lagen. Außerdem sind viele Stämme durch den Verlauf der Grenzen zu benachbarten Staaten geteilt (Zandi, Asholi, Beni Armi etc.).

Da die Grenzen meistens geometrisch und unbewacht sind, ermöglicht die freie Bevölkerungsbewegung über die Grenzen beträchtlichen Einfluß auf die nationale Identität in diesen Gebieten. Besonders bemerkbar ist dies im südlichen Teil des Sudan. Durch die 
große Entfernung von dem wirtschaftlichen und politischen Herz des Landes und durch die langjährige Flucht in die benachbarten Staaten während der Zeit der Auseinandersetzungen und des Bürgerkrieges vor 1972, erleichtert durch Gemeinsamkeiten in der Herkunft, im sozio-ökonomischen System und manchmal auch in der Sprache, sind viele Intellektuelle des Südens von Staatsideen der ostafrikanischen Länder sehr beeinflußt. Das ist in ihrem Verhalten gegenüber den vom Norden kommenden Ideen und Werten festzustellen.

Als ehemaliges Kolonialland bildet der Sudan ein seltsames Phänomen unter den afrikanischen Staaten. Nach der Eroberung ist es gemäß dem Konduminiumsvertrag vom 10. Januar 1899 unter die Herrschaft zweier Staaten geteilt worden, nämlich Großbritannien und Ägypten. Die verschiedenen Entwicklungsvorstellungen beider Kolonialmächte zeigten ihre Auswirkungen sowohl in der wirtschaftlichen als auch in der politischen Sphäre. Verbunden mit der geographischen Teilung des Sudan in den arabischen Norden und den negroiden Süden als Folge des kulturellen Nord-Süd-Wandels in Afrika, entwickelten sich schon vor der Unabhängigkeit drei politische Richtungen, die immer noch die Basis für die gegenwärtig herrschenden Ideen von nationaler Identität bilden. Diese Richtungen zielten hauptsächlich auf folgendes:

- ein unabhängiger, einheitlicher Sudan;

- ein von Großbritannien unabhängiger, mit Ägypten verbundener Sudan im Rahmen einer Einheit der Nilländer;

- ein unabhängiger Sudan mit einer Sonderstellung des Südens.

Die geographischen Gegebenheiten und die Landnutzung wirken sich auf die Identitätsfrage im Sudan unterschiedlich aus. Auf Grund der großen Fläche (mit rd. 2,5 Mio km² das größte Land Afrikas - zehnmal so groß wie die Bundesrepublik Deutschland) und der großen Nord-Süd-Ausdehnung - über 19 Breitengrade - sind verschiedene Okosysteme zu erkennen. Das Land ist normalerweise drei Hauptökosystemen zuzuordnen:

- das tropisch-subhumide Okosystem im Süden mit durchschnittlichen Niederschlagsmengen pro Jahr zwischen $900-1400 \mathrm{~mm}$ bei 6-9 humiden Monaten und natürlicher Feuchtsavannen-Vegetation;

- das nördlich anschließende tropisch-semiaride Ókosystem mit einer durchschnittlichen Jahresniederschlagsmenge zwischen $250-900 \mathrm{~mm}$ bei 2-6 humiden Monaten und mit Trocken- und Dornsavannenvegetation;

- das ganzjährig aride und extrem labile randtropische Okosystem mit einer Jahresniederschlagsmenge von 100-250 mm und der dazugehörigen Dornsavannen- bis Halbwüstenvegetation.

Eine Vielfalt verzeichnet auch das Bevölkerungspotential des Landes. Die rd. 18 Mill. Einwohner sind verschiedener Herkunft und verteilen sich auf mehr als 500 Stämme, die über 300 Sprachen sprechen. Es sind dabei Araber, Hamiten, Niloten, Nilo-Hamiten, sudanische Stämme und Negroide. Zahlenmäßig sind die Stämme verschieden groß: Sie reichen von über eine Million, wie bei den Dinka, bis zu ein paar Hundert, wie bei den Otoro in den Nuba-Bergen. Hinzu kommen auch die über zwei Millionen Menschen aus verschiedenen Stämmen Westafrikas wie Haussa, Fulami, Bornu etc., die in den Sudan 
in der jüngsten Zeitgeschichte eingewandert sind. Die Mehrheit der Bevölkerung lebt auf dem Lande. Rund 14 \% der Bevölkerung lebt in den Städten mit mehr als 8000 Einwohnern, rund $40 \%$ der übrigen $86 \%$ sind als Nomaden oder Halbnomaden klassifiziert. Daraus ergibt sich eine Vielfalt von Lebensarten, Gewohnheiten und Sozialsystemen. Die natürlichen Ressourcen, die Industrie und die Infrastruktur des Landes sind ungleichmäßig verteilt: ihre Nutzung weist eine ungerechte regionale Konzentration auf. So befinden sich im Zentrum des Landes, besonders aber am Blauen Nil, die wichtigsten und modernsten Großlandwirtschaftsprojekte und die größten Agrarindustriekomplexe des Landes. Allein auf das Gebiet der Hauptstadt, Khartoum, konzentrieren sich $70 \%$ der Industriebetriebe, $62 \%$ der Industriebeschäftigten und rd. $70 \%$ der Industrieproduktion. Obwohl in der Hauptstadt nur $6 \%$ der Bevölkerung Sudans leben, konzentrieren sich hier $40 \%$ der städtischen Bevölkerung des Landes, $90 \%$ der Hochschulplätze, die wichtigsten Erziehungs- und Bildungseinrichtungen, $50 \%$ der Ärzte, $25 \%$ aller Krankenhausbetten und $80 \%$ der Handelsgesellschaften.

\section{Regionale Identität im Sudan}

Die bisherigen Ausführungen zeigen, wie kompliziert die regionale Identitätsfrage ist. Es ist eine sehr schwierige Aufgabe, sie in ihrer Gesamtheit zu erfassen. Mit einem Vergleichsbeispiel zweier Völkergruppen bžw. Regionen im westlichen Teil des Sudan möchte ich einen Einblick in diese Problematik geben.

Die beiden Gruppen, Fur und Nuba, ähneln sich in vieler Hinsicht, aber sie unterscheiden sich auch, was wiederum den Grad der Identitätsbeeinflussung bestimmt. Die beiden Gruppen sind seßhafte Hochlandshackbauern in der semiariden Zone. Allerdings liegt der Lebensraum der Fur viel höher als der der Nuba (Jebel Marra rd. $3000 \mathrm{~m}$ hoch und Nuba Berge $1300 \mathrm{~m}$ hoch). Die Wohngebiete beider Gruppen grenzen teilweise an Räume von anderen Seßhaften, hauptsächlich aber an Gebiete nomadischer und halbnomadischer Stämme. (Abb. 1) Ostlich und westlich des Furgebietes befinden sich seßhafte Stämme, wie Masalit, Daju, Tama, Birgid und Berti. Die nomadischen und halbnomadischen Stämme sind im Norden die Zaghawa, Camel-Rigeigat und Beni Hussein. Im Süden sind verschiedene arabische Baggara-Stämme, wie Beni-Halba, Rinder-Riziegat, Taaische, Habbaniya, Missairiya etc. zu finden.

Fast an allen Seiten grenzen die Nabu an verschiedene zum größten Teil halbnomadische und nomadische arabische Stämme, wie Hawazma, Missairiya, Hamar, Beni-Hamid, Bidairiya etc. Nur im Süden sind sie den semi-seßhaften Niloten benachbart.

Obwohl die Fur und die Nomaden seit Jahrhunderten Kontakt zueinander haben, blieben sie kulturell getrennt. Unterschiede finden sich in Sprache, Lebensstil, Wirtschaftsund Sozialsystem, Siedlungs- und Haustypen, persönlicher Gebrauchsgegenstände usw. In den Nuba-Bergen dagegen, besonders im Vorland und in den Kontaktzonen, kam es zu großer Beeinflussung bzw. Vermischung der beiden Systeme. Wichtig ist in diesem Zusammenhang, daß der Kontakt zwischen Fur und Baggara gegenüber dem der Nuba- 
stämme zu den Nomaden durch die gemeinsame Religion erleichtert wird. Beide bekennen sich zum Islam; daher ist es leichter, untereinander Kontakt zu pflegen, besonders bei bestimmten islamischen Gebeten und Festen. Dagegen sind die Nuba zum größten Teil Heiden; trotzdem gehen die Auswirkungen des Kontaktes weiter. Hier treten andere Faktoren auf, wie z. B. der - im Vergleich zu den Fur - relativ weit entwickelte ErschlieBungsgrad, die höhere Anzahl von Wochenmärkten und der fortgeschrittene Urbanisierungsprozeß.

Die historische Entwicklung jeder Gruppe weist auf die Hintergründe ihres Identitätsbewußtseins hin und stellt einen wichtigen Faktor bei den Identitätsvorgängen dar. Es besteht Ungewißheit hinsichtlich der Herkunft beider Gruppen und ihrer Ansiedlung im heutigen Sudan. Obwohl die Anerkennung der Fur als einer ethnischen Einheit bei einigen Sozialwissenschaftlern umstritten ist (MacMichael 1922, Arkel 1951 u. a.), weisen die eigene, zu den sudanischen Sprachen gehörige Fur-Sprache, die gemeinsamen Gewohnheiten sowie ihre Solidarität untereinander auf eine kompakte Völkergruppe hin. Im 16. Jahrhundert entstand eine feste Organisation. Die Fur gründeten ein Sultanat, welches nicht nur die Jebel Marra-Region, sondern auch die benachbarten Gebiete umfaßte. Es gelang ihnen im 18. Jahrhundert, einen beträchtlichen Teil des heutigen Tschad und östlich alle Gebiete bis zum Weißen Nil unter ihre Herrschaft zu bringen. Dank ihrer Verbundenheit, ihrer Organisation und vor allem der Stärke des Sultan Ali Dinar war Darfur 1916 das letzte Gebiet im Sudan, das die Engländer unter ihre Kolonialherrschaft brachten - 18 Jahre nach der Wiedereroberung des Sudan. Die Fur zählen heute ungefähr 600000 Personen. Sie bewohnen hauptsächlich das vulkanische Hochland des Jebel Marra und den größten Teil der umliegenden Ebenen. Sie leben in kleinen Dörfern von 50-250 Einwohnern. Dabei sind die Siedlungsarten im Gebirge und in den Ebenen zu unterscheiden. Die Hauptunterschiede bestehen hinsichtlich der landwirtschaftlichen Fläche, des Anbau- und Bewässerungssystems.

Der Begriff "Nuba" wurde von der arabischen Stämmen für die dunkelhäutigen Bewohner der Berge in dem südlichen Teil der Provinz Kordofan verwendet. Sie zählen rd. 550000 Personen, stellen aber im Gegensatz zu den Fur keinen homogenen Stamm dar, sondern bilden eine Gruppe von über 50 Stämmen verschiedener ethnischer Herkunft, Kultur und Sprache. Bei den einzelnen Stämmen ist es ein neues Phänomen, eine Kompaktgruppe zu bilden. Bis vor kurzem waren sie in Sippen organisiert und lebten auf den einzelnen Bergen. Erst mit der Einführung des Mek-Systems durch die Engländer (indirekte Herrschaft durch Stammeshäuptlinge) und mit dem wachsenden Kontakt und Bevölkerungswanderungen kam es zur Stammesbildung bei vielen dieser Gruppen. Ähnlichkeiten und Gemeinsamkeiten zwischen diesen Teilstämmen, die sie uns als eine Gruppe betrachten lassen, sind in der gemeinsamen historischen Entwicklung der seßhaften Subsistenzwirtschaft auf der Basis von Landwirtschaft, dem gemeinsamen traditionellen Glauben, welcher auf Landwirtschaftsriten basiert und in anderen traditionellen Sozialaktivitäten und Erscheinungen, wie "Rainmaking" (Regentänze), "Sibir" (eine traditionelle Festveranstaltung - dazu noch unten), Stock-Kampf, Heiratszeremonien usw. zu sehen. 
Schon vor dem 16. Jahrhundert war die Nuba-Bevölkerung auf die Ebenen im Gebiet des heutigen Nord- und Zentralkordofan verteilt. Sie wurden in die Berge gedrängt und suchten Schutz vor Sklavenhandel, innerlichen Stammes- und Gruppenkriegen sowie den eingedrungenen nomadischen und halbnomadischen arabischen Stämmen wie Hawazma, Humer, Bedairya etc., die sich in diesem Gebiet anzusiedeln begannen. In den Bergen entwickelten die Nuba ein Lebenssystem, das der Isolierung angepaßt war. Erst Anfang dieses Jahrhunderts, nach der Eroberung des Sudan, der Errichtung eines starken Verwaltungssystems und der Beseitigung der inneren Stammeskriege begannen sie, aus den Bergen heraus in die Ebenen und Wadis zu gehen, dort Landwirtschaft zu betreiben und Siedlungen zu errichten. Dieser Prozeß der "down-hill-migration" ist immer noch im Gang und verläuft unterschiedlich von einem Stamm zum anderen. So ist der neue friedliche Kontakt mit anderen Stämmen und der Außenwelt zeitweise immer noch jung, aber seine Auswirkungen sind stärker und grundlegender als bei den Fur. Dabei spielten neben den angeführten Faktoren noch die Einführung bzw. Ausdehnung von mechanisierter Landwirtschaft, die relativ entwickelte soziale Infrastruktur und die Besonderheiten der benachbarten, bzw. der in das Gebiet eingedrungenen Bevölkerung eine große Rolle.

Im folgenden werde ich versuchen, durch den Vergleich des sozio-ökonomischen Systems beider Gruppen den Grad der Identitätsveränderung zu erläutern.

\section{Das sozio-ökonomische System der Fur}

Das wirtschaftliche System der Fur ist nach Barth (1967) in Abb. (2) dargestellt. Einige Faktoren in ihrem Arbeitsmilieu beeinflussen das ganze System. Hurst (1977) identifiziert folgendes: Größe und Struktur der Haushlte, Landeigentumsverhältnisse, Arbeitsbeschaffung bzw. -einteilung und die Wochenmärkte. Unter den Fur bildet jeder Erwachsene, männlich oder weiblich, eine unabhängige ökonomische Einheit. Auch nach der Heirat folgt die wirtschaftliche Tätigkeit den individuellen Interessen. Jeder hat seine Farm und ein eigenes Lager für seine Produkte. Dabei sind die Verhältnisse normalerweise folgendermaßen: Die Frau ist neben der Hausarbeit auch für die Kinderernährung verantwortlich, der Mann beschafft für die Familie Bekleidung und wichtige Konsumartikel vom Markt. Das Ackerland gehört formell der Sippe, aber jedes Mitglied hat das Recht auf Nutzung (Hurst 1977). Wochenmärkte sind im Jebel Marra-Gebiet gut verteilt und spielen eine große Rolle nicht nur bei der Vermarktung von Uberschuß an Hirse, sondern auch von geldbringenden Produkten, wie Zwiebeln, Knoblauch, Früchten etc. Das Geld wird wiederum benutzt für den Ankauf von Industrie- und Konsumwaren, ferner in der letzten Zeit auch für den Brautkauf (Abb. 1).

Hauptfaktor in dem System ist die Arbeitskraft der einzelnen Person, der Familie und zusätzlich dazu bezahlte Arbeitskraft Dritter (hired labour). Beim Land unterscheidet man zwischen eigenem und/oder verpachtetem Ackerland einerseits urid Kommunalland andererseits, wobei in jedem Typ eine charakteristische Landnutzung betrieben 
wird. Ackerbau bei dem ersten und Viehwirtschaft bei dem letzten. Eine entscheidende Rolle spielt auch der Einbeziehungsgrad einer Geld-Ware-Wirtschaft bei der Entscheidung für mehr Subsistenz- oder Marktproduktion.

Wie aus dem Modell hervorgeht, ist Hirse das wichtigste Anbauprodukt und wird hauptsächlich für die Deckung des eigenen Bedarfs in Form von "Asida" (Brei) und "Marisa" (Bierähnliches Getränk) verbraucht. Die Bier-Feste schaffen außerdem durch das traditionelle Nafir-System zusätzliche Arbeitskräfte. Dieses letztere, welches in vielen Arbeiten europäischer Wissenschaftler als "reciprocal labour" bezeichnet und als Bestandteil des Umtauschsystems angesehen wird, bildet einen traditionellen Wert in einer solchen Gesellschaft, der zu ihrer Identität entschieden beiträgt.

Das wird bestätigt durch die Tatsache, daß solche zusätzliche Arbeit nur in Notfällen und bei Arbeiten außerhalb der Marktproduktarbeiten herangezogen wird. Das Modell (Abb. 1) zeigt einige Alternativen im gesamten System, wobei zu bemerken ist, daß im allgemeinen die Viehwirtschaft eine untergeordnete Rolle spielt und erst nach dem Kontakt mit den Baggara-Nomaden an Bedeutung gewonnen hat. Sie bildet den wichtigsten Weg, bares Geld zu beschaffen und Kapital verfügbar zu machen. Die Größe des Kapitals wirkt sich wiederum auf das ganze System aus.

Durch verstärkten Kontakt mit Baggara-Nomaden und anderer arabischer Bevölkerung kam es zu folgenden grundlegenden Veränderungen in der Verhaltensweise und im gesamten sozio-wirtschaftlichen System:

- Durch die verstärkte Produktvermarktungstendenz entwickelte sich der bewässerte Anbau von Früchten und Gemüse. Die meisten Bewohner des Jebel Marra haben jetzt entweder einen eigenen Obstgarten oder zumindestens einige Obstbäume in der Hausfarm. Für längere Zeit hatten Zwischenhändler aus Nyala, Zalengi und El Fasher die Vermarktung dieser Produkte übernommen. Jetzt aber betreiben viele Fur den Export von Früchten selbst, sogar bis nach Khartoum und Port Sudan, über eine Entfernung von rd. $2000 \mathrm{~km}$. Das wiederum führt zu neuen Kontakten und Informationsmöglichkeiten.

- Die wachsende Tendenz zu verstärkter Viehwirtschaft als der wichtigsten Quelle für die Kapitalansammlung wurde schon erwähnt. Diese aber brachte grundlegende Veränderungen und Adaptionen im sozio-ökonomischen System mit sich. Die Fur mußten schnell erkennen, daß sie verschiedene Naturpotentialzonen mit saisonaler Grasdecke brauchten, um solche Wirtschaftsform entsprechend ihrem Arbeitsmilieu den ökologischen Voraussetzungen verwirklichen zu können. So wurden die ersten Einflüsse des Kontakts mit den Nomaden spürbar. Viele Fur-Bauern überließen zuerst ihr Vieh den Baggara-Nomaden nach traditionellen Vereinbarungen. Dabei hat der Baggara-Hirte keine Verantwortung für Krankheiten oder Todesfälle der Tiere. Solche Arrangements haben deshalb Nachteile, gelten nur als Vertrauenssache und finden keine rechtliche Anerkennung. Einerseits aus Mißtrauen und andererseits wegen der Weigerung von Baggara-Nomaden, viele Tiere mitzunehmen, haben einige Fur begonnen, die Wanderung selbst durchzuführen, sind also zum Nomadentum übergegangen. Dieser Prozeß verstärkte sich in den letzten Jahren, da die Tierpreise wegen 
wachsender Exporte in benachbarte Olländer und steigenden Fleischverbrauchs in den Städten um ein Vielfaches gestiegen sind.

Die Umwandlung von seßhaften Bauern zu Nomaden oder Halbnomaden hat dennoch die Identität der Fur nur wenig beeinflußt. In einigen Fällen führte sie sogar zur verstärkten Identitätsfindung bzw. -äußerung. Die Fur machen die Wanderung gemeinsam mit den Baggara-Nomaden, zelten mit auf ihren Lagerplätzen, aber trotzdem unterscheiden sie sich von den Baggara. Sie versuchen, möglichst im Fur-Gebiet zu bleiben, d. h. kurze Wanderungen zwischen Berg und Ebenen bzw. Jebel-Qoz-Vorland zu machen. Dabei halten sie sich zusammen: Sie zelten im Lager nebeneinander und reden unter sich in der Fur-Sprache. Ein Vorteil für sie ist, daß sie sich nicht weit von ihren Dörfern und Anbaugebieten entfernen und zugleich während der Regenzeit Landwirtschaft betreiben können. Nur die Fur mit großen Tierhaushalten machen die langen Wanderungen in Baggara-Gebiete mit und verzichten dabei auf Hirseertrag aus ihrem eigenen Land, da das Vieh mehr Geld einbringt. Auch bei diesen langen Wanderungen verhalten sich die Fur wie eine Minderheit; sie behalten ihre kulturelle Identität, versuchen ihren Kindern, die mit den Baggara-Kindern aufwachsen, neben der arabischen auch die FurSprache beizubringen und veranstalten ihre Heirats- und anderen Feste nach der FurTradition.

Der Unterschied zwischen beiden Gruppen besteht hier auch darin, daß der Anreiz bei den Fur nur Geld ist, bei den Baggara aber eine traditionelle Gewohnheit. Dieser Kontakt verläuft nicht spurlos. Verstärkter arabischer Einfluß zeigt sich durch wachsende Benutzung der arabischen Sprache, Veränderungen in Eß- und Nahrungsgewohnheiten. Am wichtigsten ist, daß sich dadurch eine neue Arbeitsteilung innerhalb der Familie als Folge der Struktur dieser neuen Wirtschaftsform ergibt. Statt getrennte Felder und Lagerräume erfordern hier die verschiedenen Tätigkeiten dieser Landnutzungsform die gemeinsame Arbeit aller Familienmitglieder; sogar die Kinder sind bereits bei der Wasserbeschaffung miteinbezogen. Andere Auslöser der Identitätsveränderung bilden u. a. die urbanen Zentren, die Wochenmärkte, Schulen und verschiedene Informationsmedien. Man merkt ihren Einfluß z. B. an größerer Verbreitung der arabischen Sprache und an Bekleidungs- und Eßgewohnheiten aus dem Nord- und Zentralsudan. Veränderungen sind auch in Hausbau und Siedlungsstruktur zu sehen. Normalerweise leben die Fur in unverschlossenen Einzelhütten ohne Zäune. In einigen Siedlungen, besonders in größeren Dörfern, sind heute Zäune vorhanden, und die Häuser werden verschlossen, wie es in den großen Siedlungen des Zentralsudan üblich ist.

\section{Das Wirtschaftssystem der Nuba und die Identitätsf rage}

Das ökonomische System der Nuba unterscheidet sich vom Fur-System in mehreren Bereichen (Abb. 2): -

Erstens bildet die Familie eine wirtschaftliche Einheit, obwohl manchmal Mann und Frau getrennte Felder bearbeiten. Es handelt sich hier um eine rein organisatorische 
Frage in der Arbeitsteilung. Da die Frau noch dazu im Haus arbeitet und die Familie betreut, betreibt sie ihre Hauptanbautätigkeit auf der kleinen naheliegenden Hausfarm, und der Mann bestellt das weitentfernte Feld. Sie leistet ihm auch dabei Hilfe, besonders bei der Ernte, da Anbau und Ernte beider Typen zu verschiedenen Zeiten geschehen. Zweitens spielt die Vermarktung von Produktion und dabei auch die Geldbeschaffung hier eine größere Rolle, da man bares Geld nicht nur für den Kauf von Konsum- und Industriewaren braucht, sondern davon auch die gesetzlich geforderten Landwirtschaftssteuern zahlen muß (Abb. 2).

Drittens steht hier durch zeitweilige Arbeit in großen und naheliegenden Urlaubszentren mehr Geld zur Verfügung. Viele junge Nuba-Männer wandern jährlich für bestimmte Zeit in die Städte und nehmen gelegentliche Arbeit in der Industrie, im Dienstleistungssektor und als Hausdiener auf. Sie kehren vor der Regenzeit zurück, um Landwirtschaft zu treiben und das Familienleben zu genießen. Das wirkt sich wiederum auf das ganze System aus.

Viertens ist die Viehwirtschaft hier eine alte Tradition und bedeutender für die Kapitalvermehrung und das Sozialleben. Mehr Fleisch wird - abgesehen von den Marisa-Festen im Kujursystem - auch in den traditionellen Sibir-, Trauer- und Heiratsfesten verbraucht.

Fünftens bieten sich in diesem System mehr Alternativen. Wegen der angeführten Faktoren: Durch den Verkauf von Brennholz und Holzkohle von den relativ stark bewaldeten Flächen und durch die Existenz vieler Städte, Großdörfer und mechanisierter Großlandwirtschaftsbetriebe gibt es hier andere Wirtschaftsquellen.

Die Lage der Nuba-Berge in der Nähe des wirtschaftlich bedeutenden Zentrums des Landes und die daraus resultierenden Faktoren vertieften den Kontakt der Nuba mit anderen Gesellschaften außer den benachbarten arabischen nomadischen und halbnomadischen Stämmen. Die Erschließung des Gebietes durch relativ bessere Verkehrsstraßen und -pisten erlaubte es vielen Kaufleuten und anderen aus dem Nord- und Zentralsudan, sich in diesem Gebiet anzusiedeln. Schon während der letzten Jahrzehnte der Kolonialherrschaft hat man das bedeutende Naturpotential dieser Gebiete entdeckt, welches in dem fruchtbaren, für Landwirtschaft geeigneten Boden liegt. Es handelt sich um einen lehmigen, erheblich mineralreichen Boden, als "Gardud" bezeichnet, und einen tiefbraunen Lehmboden, "Cotton Soil" genannt. Letzterer zeigt die Ausdehnung der "General Clay Plains" im Zentralsudan, zu denen die anderen wichtigsten Landwirtschaftsprojekte wie Gezira, Rahad und mechanisierter Anbau bei Gedaref gehören. Deshalb führte man den Anbau von Baumwolle in dieses Gebiet ein. Die Anbaufläche hat sich seit der Unabhängigkeit stark ausgedehnt, allerdings mit zunehmender Orientierung auf Hirse und Olsaaten, wie Sesam und Erdnüsse. Neue Ausdehnungen dieses mechanisierten Anbaus in der Region der Nuba-Berge werden u. a. von der Bundesrepublik Deutschland finanziert.

Die wirtschaftliche und die damit verbundene verkehrsmäßige und industrielle Erschließung des Raumes verursachten große Wanderungen in das Gebiet, die auch nicht geschlossene Stammesabteilungen umfaßten. So kamen aus dem nördlichen und Zentral- 
sudan Händler (als Gallaba bezeichnet), Großbauern, Lehrer und Regierungsbeamte. Innerhalb des Einwanderungsprozesses der Westafrikaner in den Sudan bildeten diese Gebiete wichtige Ziele für die Fulani, Haussa und Borno-Stämme aus Nordnigeria, Kamerun und Tschad. So entstanden viele große bäuerliche Siedlungen, die sich rasch in Städte, Baumwollmärkte und Dienstleistungszentren entwickelten. Beispiele dafür sind Kadugli, Dilleng, Heiban, Abu Gubieha und Abbasiya.

Durch die ökologische Degradation in den nördlichen Regionen der Provinz Kordofan verstärkte sich die Wanderung der Nomaden in die Nuba-Berge. Neue nomadische Stämme, sogar Kamelnomaden, haben seit Anfang der siebziger Jahre begonnen, dieses Gebiet regelmäßig zu besuchen. Während eines Untersuchungsaufenthaltes im Sommer 1980 fanden wir in der Dorfschule von Kalkada - im südlichen Teil der Nuba-Berge - sogar 30 Schüler aus dem Maganin-Stamm, dessen Mitglieder Kamelzüchter sind.

Alles das ermöglicht den Nuba mehr Kontakt mit verschiedenen Kulturen und Lebensarten, was sich wiederum auf ihre Identität auswirkt. Die daraus resultierenden Modifikationen im Lebenssystem sind im folgenden aufgeführt:

- Mit dem wachsenden Prozeß der "down-hill-migration" und damit einer für andere Stämme zugänglichen Siedlungsweise ist intensive Orientierung und Interesse an Marktproduktion und damit eine vertiefte Einbeziehung in eine Geld-Ware-Wirtschaft zu verzeichnen. Das bedeutet auch, daß man mehr produziert, um Úberschuß zu vermarkten und Geld zu beschaffen. Dabei ist aber eine alte Tradition verschwunden, nämlich aus Sicherheit den Uberschuß der guten Jahre für die schlechten Erntejahre zu lagern. Man verkauft jetzt den Ưberschuß, um in den Jahren mit einem geringeren Ernteertrag - aus Regenmangel oder aus anderen Gründen - für vielfaches Geld Hirse aus dem Markt zu beziehen.

- Die alten Eigentumsverhältnisse und das traditionelle Erbsystem wurden dabei kaum geändert. Aber Wertbewußtsein in bezug auf die landwirtschaftliche Fläche entwikkelte sich - so holzte man größere Waldflächen ab, nahm sie als Eigentum in Anspruch oder verpachtete sie weiter.

- Die islamische Religion und die arabische Sprache verbreiteten sich schnell, besonders in Kontaktzonen. In diesen Gebieten haben die Nuba viele Traditionen von den arabischen nomadischen und seßhaften Stämmen übernommen. Ein großer Teil der neuen Generation in den Urbanzentren beherrscht nicht mehr die eigene Sprache. Es muß andererseits erwähnt werden, daß, obwohl einige Nuba die islamische Religion und die arabische Sprache übernommen haben, sie immer noch etwas von ihrer eigenen Tradition und ihrem Glauben behalten. Sie verhalten sich manchmal unislamisch. So ist der Glaube an "Kujur"(Zaubermediziner) und das Sibir-System immer noch verbreitet und gebräuchlich. Bei "Sibir" handelt es sich um eine Festveranstaltung - eine Mischung aus Zauber und Glauben - durchgeführt bei Krankheit, verspätetem Regen, Konflikten und Zukunftsvoraussagen. Dabei nehmen neben der betreffenden Person alte Männer oder Kujur teil. Der Veranstalter muß einen Bock und einen Hahn opfern, die während des Festes geschlachtet werden.

- Große Veränderungen folgten auch in vielen Gewohnheiten ihres täglichen Lebens. 
So entwickelte sich hier die im Norden und in den Urbanzentren verbreitete Weizenund Hirsefladenbrotherstellung. Bei vielen Häusern setzten sich Zäune, Rakubas (Vorzimmer) und Toiletten durch. Die schnelle Verbreitung von arabischer und europäischer Schlagermusik sowie Folklore und Tanzarten bestimmter Gruppen im Nord- und Zentralsudan ist vielleicht ein Beweis dafür, welch geringe Resistenz die Nuba gegenüber anderen Kulturen haben. Das zeigt auch der große Einfluß der Gallaba (Kaufleute aus Nordsudan), jugendliche Rückwanderer aus Urbanzentren, Informationsmedien und Lastkraftfahrern. Besonders sind die Rückwanderer für die Verbreitung von Transistorradios, Tonbandgeräten, westlichen Bekleidungen und Metallmöbeln verantwortlich.

- Veränderungen durch den Kontakt mit Nomaden sind noch an einer anderen Stelle zu bemerken. Obwohl die Viehwirtschaft bei den Nuba eine alte Tradition ist, kannten sie keine großen Wanderungen. Sie bewegten sich zwischen Berg und Ebenen. Heute aber übernehmen viele Nuba die Baggara-Art von großen Wanderungen, die während der Trockenzeit bis zum Bahr el Arab im Süden reichen. Die früheren, kleinen Tiere der Nuba sind durch Baggara-Sorten ersetzt, und die Zahl der Herden hat sich vergrößert. Gegenüber den nomadischen Fur versuchen die Nuba nicht, in Zeltplätzen erkennbare Gruppen zu bilden.

\section{Folgerungen}

In multi-ethnischen Staaten Afrikas, wie dem Sudan, spielt die Frage der Identität eine besondere Rolle. Während der Kolonialzeit hat ein Prozeß kontrollierter Entfremdung stattgefunden, wobei die politische, soziale und wirtschaftliche Entwicklung durch europäische Vorbilder beeinflußt wurde. Nach der politischen Unabhängigkeit hat eine Gegenbewegung begonnen, gekennzeichnet durch die Suche nach einer eigenen Identität, speziell im sozialen und kulturellen Sinne. Dadurch hoffen diese Völker, sich zu entdekken und zu stärken. Allerdings verläuft dieser Prozeß nicht ohne Schwierigkeiten. Es überschneiden sich nationale regionale und Stammesinteressen. In diesem Prozeß lösen sich einige schwache kulturelle und soziale Besonderheiten auf, andere aber verstärken sich und tragen zur Verstärkung der Identitätsfindung bzw. -äußerung bei. Dieser Fragenkomplex ist auch von besonderer Bedeutung für die Geographie: Diese Länder sind nicht nur von wirtschaftlichen und allgemein politischen Interesse, sondern auch Räume der Identifikation. Der regionale Vergleich am Beispiel zweier Völkergruppen im Sudan - Fur und Nuba - zeigt, wie der Prozeß der Identitätsfindung, -erhaltung und ihres Verlustes vom geographischen Faktoren beeinflußt wird. Dies kann aber nur als allgemeine Einschätzung gelten; weitere Erforschung seitens der Geographie und anderer sozialer Disziplinen ist notwendig. 


\section{Literatur:}

Arkell, A. J., (1951): "History of Darfur A. D. 1200-1700" Sudan Notes and Records, Khartoum, 1951.

Babiker, A. B . A. (1974): "Grundlagen der Entwicklung und Standortverteilung der Industrie im Sudan" MartinLuther-Univ., Halle (Diss. - unveröffentlicht).

Babiker, A. B. A, Shaddad, M. Z., Musnad, H. (1981): "Wood resource use in Nuba Mountains" Forschungsarbeit der UNO-Univ., Tokyo (Unveröv.).

Barbour, K. M. (1961): "The Republic of the Sudan - a regional geography" - London 1961.

Barth, F. (1970): "Economic groups and boundaries: the social organization of culture diff erence" London, 1970.

Hurst, M. E. (1972): "A Geography of Economic Behaviour" Calif., 1972.

Ibrahim, F. H. (1980): "Desertification in Nord-Darfur" Hamburger Geogr. Studien, Hamburg, 1980.

Mac Micheal, H. A. (1922): "A history of Arabs in the Sudan" Cambridge, 1922.

Mensching, H. (1978): "Zur Geomorphologie des J. Marra - Darfur/Sudan“, Beiträge zur Quartär- und Landschaftsforschung - Wien, 1978.

Roden, D. (1972): "Down-migration in the Moro-Hills of S. Kordofan", Sudan Notes and Records, Khartoum, 1972.

Stevenson (1962): "Linguistic Research in the Nuba Mountains" Sudan Notes and Records, Khartoum, 1962.

Tessler, M. u. a. (1973): "Tradition and Identity in Changing Africa" - London, 1973. 


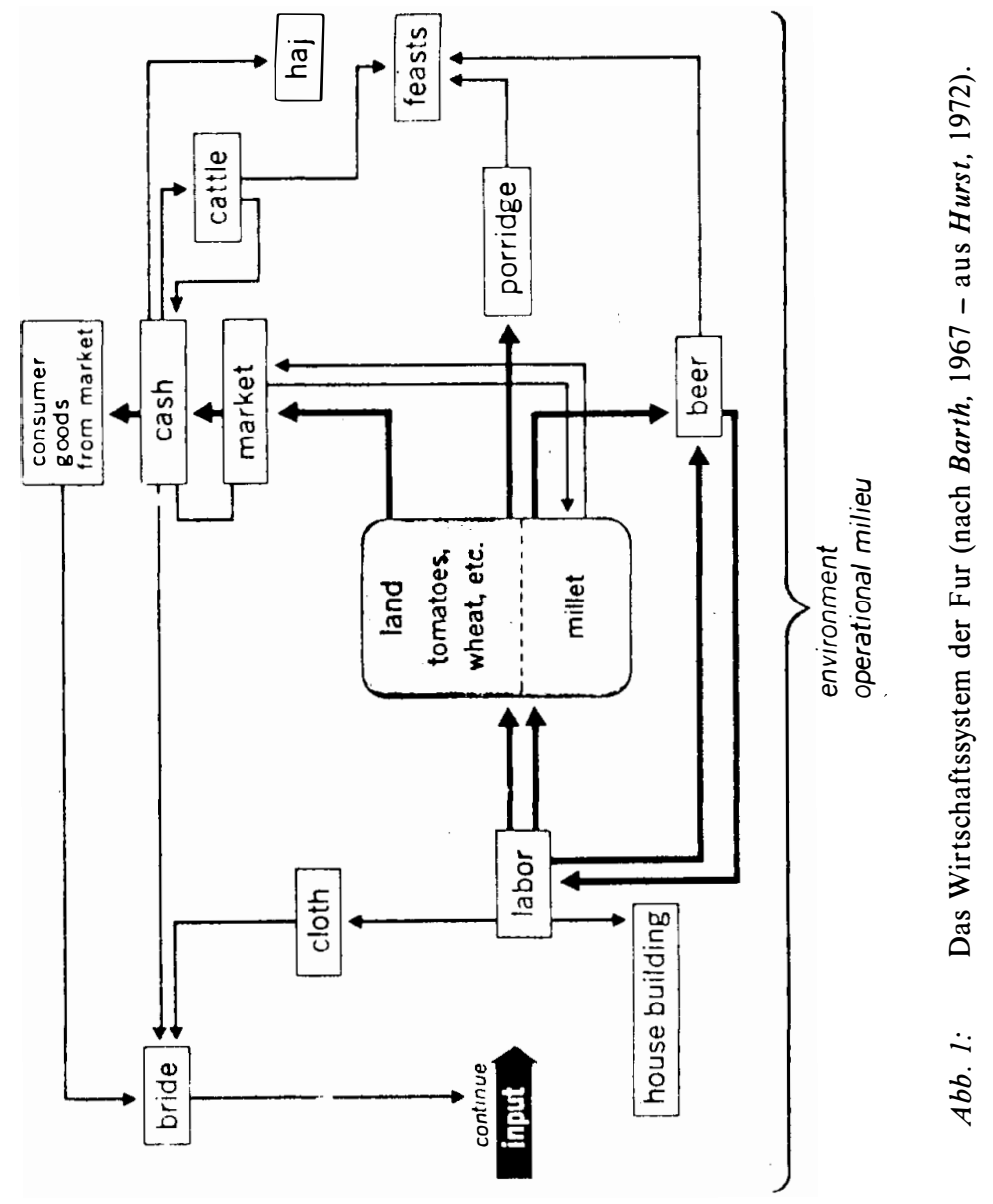




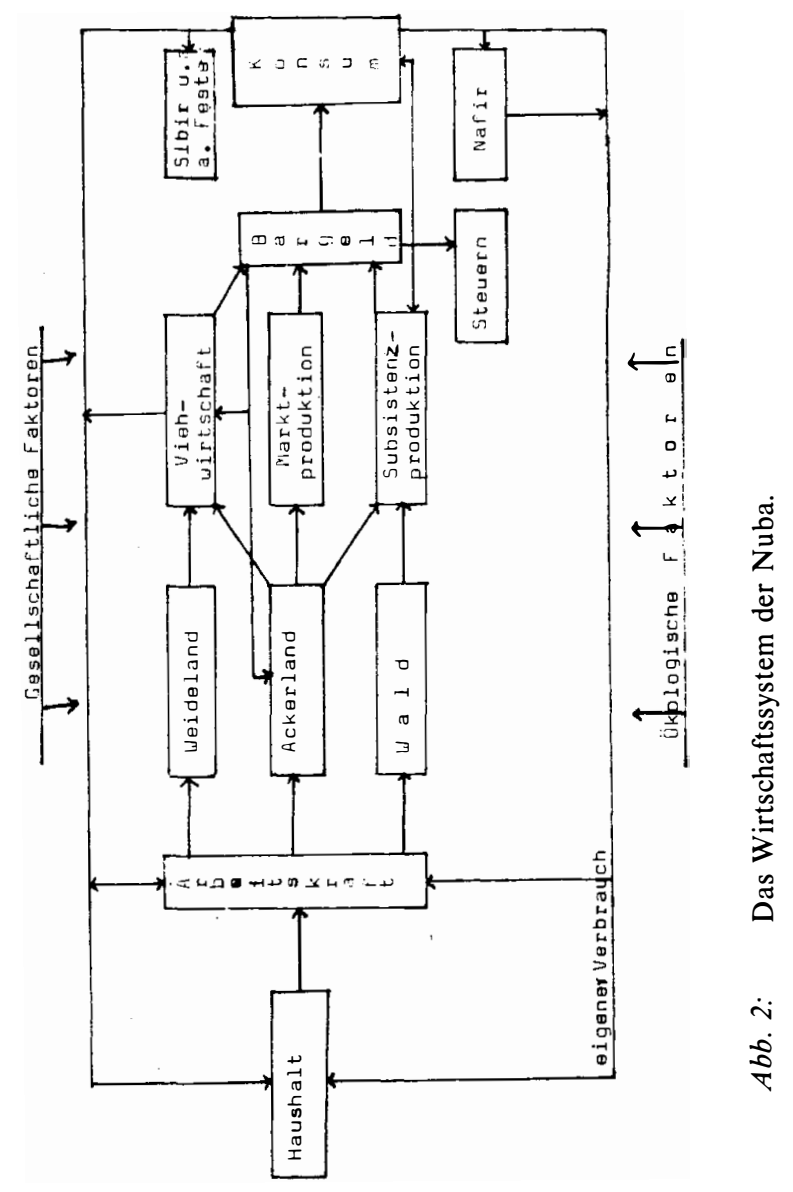




\title{
ABSTRACTS
}

\section{Political Justice in Kenya: Prolegomena to an Inquiry into the Use of Legal Procedures for Political Purposes in Post-Kenyatta Era}

\author{
By O. Ooko-Ombaka
}

This study analyses the use of judicial and administrative procedures by the Moi government to consolidate its succession to power following Kenyatta's death. This is the phenomenon termed political justice.

It is divided into two broad sections. The first section on the political economy of political justice provides the theoretical superstructure of the study. It is argued that the degree of political justice is inversely proportional to the political power and confidence of the ruling class. The increased reliance on judicial and administrative procedures by the Moi government is thus located in its relative weakness. The second section, examines through case law, different levels of political justice, namely: the criminalization of politics, cases involving direct attack on established constitutional order, political prosecutions and the use of judicial procedures to subvert the democratic electoral process. The conclusion evaluates the impact of political justice suggesting lines for possible further research.

\section{Problems of National and Regional Identity in Sudan}

\section{By Abdel Bagi A. G. Babiker}

The question of identification plays a particular role in multi-ethnical African states. Racial, regional and national interests are overlapping and their interaction is influenced by the social position of the different groups within the hierarchy system. The attitude of group members vis-a-vis society is changing as far as moral, traditional and cultural identity is concerned, this being a result of intensified relations with other cultures through the media and of the process of urbanization.

On the national level regional particularities often almost disappear when periphery. identity is adapted to center identity. On the other hand regional identity can become stronger by the research for identity.

As a country characterized by a large territory as well as ethnical and ecological plurality Sudan is a good example for such ambiguity. By comparing two ethnical groups in the western part of the country - Fur and Nuba - we can observe how the process of fin- 
ding, preserving and loosing identity is influenced by geographical factors. Although both groups are similar in many respects, the different socio-economical systems deeply influence the degree of change in identity.

\section{The interaction of agrarian movements and political changes in India}

\section{By Theodor Bergmann}

The introduction stresses the role of the peasantry in the shaping of history. - Thereafter, some functional definitions of agrarian movements are offered. They express the basic social needs of the rural masses in a countryside, which is clearly stratified and rent by social tensions and struggles: land, irrigation, the right to dispose of the produce. In a feudal society, the landlords are the given enemies of the cultivators. But even after the feudal order is removed, the shortterm interests of the peasantry might clash with the objectives of society. - Agrarian movements are manifold and take many forms; they are distinct from social movements of the urban proletariat by several traits. The delimitation of the rural social strata is diffuse, and there exist clientele relationships across the strata.

Agrarian movements have had an important share in shaping the political development of India from the start of the independence movement in the 1880's to the split of the Congress party in the late 1960's. Several researchers have tried to build a theoretical framework and to offer analyses of the many local and regional movements and their impact on the nation as a whole.

The Congress was in fact split on the approach to the rural poor. Nehru was willing to use their strength fully and to remunerate them after independence by a thorough agrarian reform. Gandhi and his later disciple Vinoba Bhave hesitated in face of these uncontrollable forces. This was also the cause of the final failure of Bhave's land-gift movement.

Legal agrarian reform was successful in Kerala and West Bengal, where there was an intensive interaction and mutual support of peasant movement from below and radical state government from above. In most other states land reform was abortive. - The radical Telengana movement, on the other hand, was widespread and created a parallel "soviet" government in large parts of the erstwhile state of Hyderabad. Though it was quelled brutally, it triggered off the demise of the Nizam of Hyderabad, the integration of the state in the Indian union, Bhave's land-gift movement, the administrative reform of India and a legal land-reform in the state of Andhra Pradesh in the 1970's.

In spite of defeats, new movements emerge again and again, e.g.the naxalites, the landgrabbing movement in the early 1970's, and heavy struggles between landlords and cultivators are frequently reported, leading to convulsions in state and central politics.

The peasant movements are largely dominated and led by the two main communist par- 